\title{
ENERGY BALANCE IN SEALED ELECTRIC TECHNICAL SYSTEMS FOR SPECIAL APPLICATIONS
}
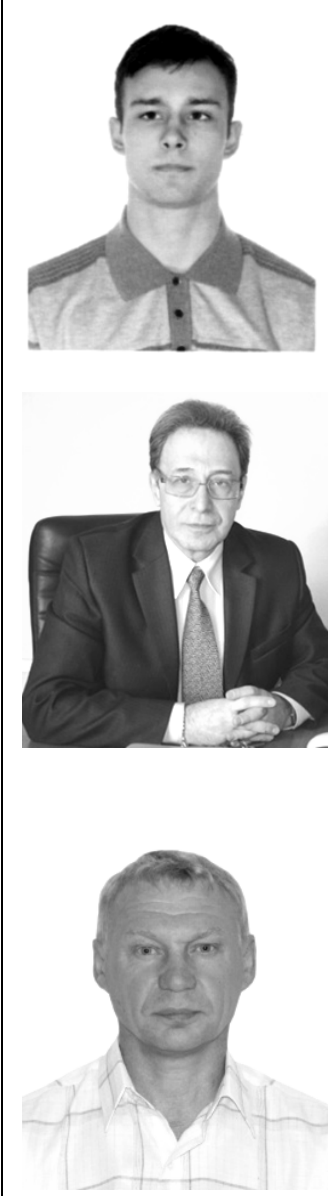

Голоколос Дмитрий Анатольевич - аспирант кафедры «Электромеханика» Комсомольского-на-Амуре государственного технического университета (Россия, Комсомольск-на-Амуре); 681024, г. Комсомольск-на-Амуре, пр. Интернациональный, 59 - 31; +7 (914) 2123472. E-mail: dag@land.ru.

Mr. Dmitry A. Golokolos - PhD candidate, Department of Electromechanics, Komsomolsk-on-Amur State Technical University (Russia, Komsomolsk-on-Amur); 681024, Komsomolsk-on-Amur, 59, Internatsionalny Av., 31; +7-914-2123472. E-mail: dag@land.ru.

Гринфельд Григорий Михайлович - кандидат технических наук, доцент кафедры «Электропривод и автоматизация промышленных установок и технологических комплексов» Комсомольского-на-Амуре государственного технического университета (Россия, Комсомольск-на-Амуре); 681018, г. Комсомольск-наАмуре, ул. Пионерская, 61 - 20; +7 (914) 1743914. E-mail: grinfelds2002@mail.ru.

Mr. Grigory M. Grinfeld - PhD in Engineering, Associate Professor, Department of Electric Drive and Industrial Automation, Komsomolsk-on-Amur State Technical University (Russia, Komsomolsk-on-Amur); 681018, Komsomolsk-on-Amur, 61, Pionerskaya Str., 20; +7-914-1743914. E-mail:grinfelds2002@mail.ru.

Иванов Сергей Николаевич - доктор технических наук, доцент, профессор кафедры «Электромеханика» Комсомольского-на-Амуре государственного технического университета (Россия, Комсомольск-на-Амуре); 681021, г. Комсомольск-на-Амуре, ул. Котовского, 1 - 60; 8 (914) 1859120. E-mail: isn@initkms.ru. Mr. Sergey N. Ivanov - Doctor of Engineering, Professor, Department of Electromechanics, Komsomolsk-on-Amur State Technical University (Russia, Komsomolskon-Amur); 681021, Komsomolsk-on-Amur, 1, Kotovsky Str., 60; +7-914-1859120. E-mail: isn@initkms.ru.

Аннотация. Расширение областей применения электротехнических комплексов предполагает изменение подходов к обоснованному выбору энергетических соотношений на стадии проектирования. В данной статье рассмотрены вопросы определения соотношений между механической и тепловой составляющими мощности герметичных электротехнических комплексов специального назначения. Целью исследования является получение расчетных выражений, позволяющих не только оценивать, но и обеспечивать требуемые энергетические соотношения в рассмотренном классе устройств.

Summary. Expanding the fields of application of electro technical systems implies changing our approaches to the sound choice - at the design stage - of energy relations. In the present paper we tackle the problem of how to define the relations between mechanical and heat components of the power of pressure-proof specialized electro technical systems. The goal of our investigation is to obtain design-basis expressions allowing us to both assess and ensure the required energy relations in the systems of this class.

Ключевые слова: герметичные электротехнические комплексы, охлаждающая жидкость, теплообмен.

Key-words: pressure-proof electro technical systems, coolant, heat exchange. 


\section{Учёные записки \\ Комсомольского-на-Амуре государственного технического университета}

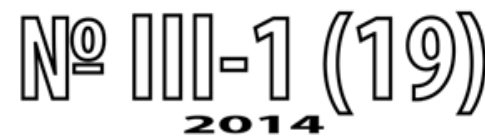

В течение длительного времени ведущими производителями электротехнических комплексов и систем для самых разнообразных областей применения велись разработки стандартного энергетического оборудования повышенной производительности с высокими эксплуатационными свойствами. При этом на практике часто имеет место необходимость в устройствах, одновременно соответствующих жестким требованиям стандартов (например, IEEE 841-2009, DIN EN ISO 9001, ГОСТ РВ 0015-002-2012) и работающих в условиях, существенно отличающихся от номинальных. Примерами такого оборудования являются широко используемые приводы с жидкостным охлаждением, электротехнические установки, подключаемые к сети с отклонениями амплитуды, формы и частоты напряжения питания, теплообменные устройства со сниженным уровнем потерь на прокачку теплоносителя, виброустойчивые электромеханические преобразователи и в том числе герметичные электротехнические комплексы специального назначения. Наибольший интерес в этой области представляют разработки фирм Сименс и Франклин Электрик, использующих электроприводы в герметичной оболочке для мощных компрессоров и насосов, нашедшие дальнейшее развитие в отечественных герметичных электромеханических преобразователях [1].

В тоже время анализ и оценка влияния конструкционных элементов на эксплуатационные характеристики герметичных электротехнических комплексов (ГЭК) показывают, что при их работе имеют место дополнительные потери в экранирующих элементах, в основном обратно пропорциональные удельному электрическому сопротивлению материала. Значимость электрических свойств экранов подтверждают результаты расчетов: при одинаковом значении индукции потери для равнотолщинных экранов из нержавеющей стали и железа, т.е. при изменении удельного электрического сопротивления от $1,150 \cdot 10^{-6}$ Ом·м до $0,098 \cdot 10^{-6}$ Ом $\cdot$ м, отличаются почти на порядок [1]. Относительные потери могут составлять от $6 \ldots 8$ \% (для маломагнитного экрана из нержавеющей стали) до 70...80 \% (для ферромагнитного экрана), что указывает на необходимость повышения эффективности системы отвода выделяемой в элементах тепловой мощности и, как следствие, на целесообразность ее утилизации, например в ГЭК, в варьируемой степени совмещающих функции генерации и транспортирования тепловой энергии [2]. Причем именно количественное соотношение между обеспечиваемыми при эксплуатации тепловыми и механическими мощностями определяет назначение электротехнического комплекса (электронагревательный, перекачивающий или совмещенный для одновременного нагрева и перемещения рабочей среды) [3].

Таким образом, задача разработки и проектирования ГЭК заданного целевого назначения в первую очередь требует определения энергетических соотношений между тепловой мощностью, отводимой охлаждающей жидкостью и механической мощностью, обеспечивающей перемещение этой жидкости относительно герметизирующего экрана, и сводится к нахождению электромагнитных и геометрических параметров, соответствующих требуемым величинам выходной температуры и производительности (расхода) или давления (напора). Очевидно, что для эффективного решения и нахождения оптимальных проектных параметров необходимы не только математическое описание физических процессов в ГЭК и численное моделирование на основе мультифизичных программ (ANSYS, FEMLAB, FLUENT, FLUX, OPERA, FLOWVISION, ELCUT), но и многомерная параметризация объекта исследования с использованием возможностей САПР-технологий (SOLIDWORKS, T-FLEX CAD и аналогичных САПР), результаты которых могут быть использованы для качественной и количественной оценки процесса нагрева и транспортирования.

Однако уже на этапе постановки задачи исследования возникает необходимость в задании начальных и граничных условий, которые априори не могут быть определены из-за сложности распределения элементов потока охлаждающей жидкости, обусловленной неравномерностью профиля скоростей потока, его турбулентностью, образованием не учитываемых разрывных течений, застойных зон, циркуляций, температурных градиентов и связанных с ними течений, междуфазовыми процессами тепло- и массообмена и т.п. 


\section{Учёные записки \\ Комсомольского-на-Амуре государственного технического университета}

Как классическая (объемно-элементная), так и диффузионная модели не описывают реальный режим течения охлаждающей среды в ГЭК. Анализ гидродинамических условий, применительно к конструктивному исполнению вращающихся элементов совмещенных электротехнических комплексов, показывает, что для них могут быть использованы смешанные (комбинированные) модели, описывающие совокупность взаимосвязанных областей потока с различными характеристиками. Задача синтеза такой модели для различных расчетных областей как для простых идеализированных режимов течения (смешения, вытеснения, вытеснения с диффузией), так и для сложных (частично несмешиваемых, обратноциркуляционных, струйных потоков) включает этапы предварительного определения конфигураций и объемов зон с различными типами течения жидкости, например на основе теории пограничного слоя и параметризации модели, одновременно обеспечивающей точность отображения тепловых и гидравлических процессов и ограничивающей сложность математических представлений, необходимых для ее анализа.

Идентификация характерных целевых областей тепло- и массообмена, условно показанная на рис. 1, может быть произведена на основе анализа конструкций идеального смешивания (интенсивность циркуляции потока приводит к равномерному перемешиванию с радиальной скоростью $v_{r}$ поступающей и уже находящейся в апертуре жидкости) и идеального вытеснения (поток состоит из равномерно движущихся с одинаковой осевой скоростью $v_{z}$ частиц жидкости).

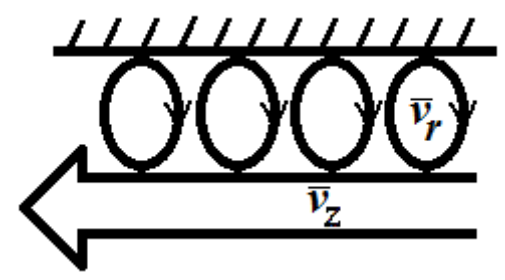

Рис. 1. Комбинированная модель тепло- и массообмена

Основное условие применения модели идеального смешивания - требуемая интенсивность перемешивания - обеспечивается автоматически вследствие наличия механического перемешивающего исполнительного элемента. Для модели идеального вытеснения, предполагающей поршневой характер течения охлаждающей среды, перемешивание потока отсутствует и время движения жидкости равно отношению внутреннего объема ГЭК к объемному расходу, например как в трубчатых теплообменных аппаратах при турбулентном течении жидкости с равномерным профилем скорости. Параметризация модели сводится к нахождению значимых факторов и функционала, однозначно описывающего выходные характеристики и целевое назначение ГЭК [4]. Комбинированная модель позволяет рассматривать два условно независимых процесса (нагрев и перемещение охлаждающей среды) и, соответственно, установить энергетические соотношения в ГЭК. При этом процесс перемещения является основным, поскольку определяет как интенсивность теплоотвода от герметизирующей оболочки, так и производительность комплекса.

На этапе определения энергетических соотношений, характеризующих явления массопереноса в ГЭК, целесообразно предварительно воспользоваться моделью идеализированного однопоточного и одноступенчатого источника механической мощности, имеющего бесконечно большое число плоских лопастей единичной толщины, перемещающих физическую среду, а затем провести параметризацию и учесть гидравлические, объемные и механические потери. Такой модели соответствует конструкция в виде совокупности двух торцевых колец, соединяющих напорные лопасти, причем при параметризации учитываются число лопастей, их геометрические размеры, профиль, углы входа/выхода потока.

Используемая в гидравлике формула Эйлера в виде разницы скалярных произведений $\boldsymbol{u}$ и $\boldsymbol{v}$, записанная для системы лопастей, вращающихся относительно продольной оси с частотой $n$, имеет следующий вид: 


\section{Учёные записки \\ Комсомольского-на-Амуре государственного технического университета

$$
g H=\left(u_{2}, v_{2}\right)-\left(u_{1}, v_{1}\right),
$$

где $g H$ - удельная энергия, передаваемая исполнительным элементом с бесконечно большим числом лопастей несжимаемой жидкостью без учета нагрева; $v_{2}$ - абсолютная скорость охлаждающей жидкости на выходе; $v_{1}$ - абсолютная скорость охлаждающей жидкости на входе.

Левая часть уравнения (1) может быть раскрыта с учетом профилей лопастей исполнительного элемента:

$$
g H=\left(u_{2} \sin \gamma_{2}-u_{1} \sin \gamma_{1}\right)\left(u_{2} \sin \gamma_{2}+u_{1} \sin \gamma_{1}\right)-\left(u_{2} v_{2 r} \sin ^{2} \gamma_{2} \operatorname{ctg}\left(\pi-\beta_{2}\right)-u_{1} v_{1 r} \sin ^{2} \gamma_{1} \operatorname{ctg} \beta_{1}\right),
$$

где $\gamma_{2}$ - угол скоса внешней кромки лопасти; $\gamma_{1}$ - угол скоса нижней кромки лопасти; $v_{2 r}-$ радиальная составляющая абсолютной скорости охлаждающей жидкости на выходе; $v_{1 r}-$ радиальная составляющая абсолютной скорости охлаждающей жидкости на входе; $\beta_{2}-$ угол установки лопасти на выходе; $\beta_{1}$ - угол установки лопасти на входе.

Выходные и входные тангенциальные и радиальные составляющие абсолютной скорости определяются по выражениям

$$
\begin{gathered}
u_{1}=\pi D_{1} n, \\
u_{2}=\pi D_{2} n, \\
v_{1 r}=\frac{Q}{\pi D_{1} b_{1}}, \\
v_{2 r}=\frac{Q}{\pi D_{2} b_{2}} .
\end{gathered}
$$

где $D_{1}$ - теоретический диаметр, образованный внутренними кромками лопастей; $D_{2}$ - теоретический диаметр, образованный наружными кромками лопастей; $b_{1}$ - ширина лопасти на входе потока; $b_{2}$ - ширина лопасти на выходе потока; $Q$ - производительность.

Подстановка выражений (3) в формулу (2) позволяет получить уравнение, связывающее давление $H$, создаваемое перемещаемой жидкостью, и производительность системы охлаждения:

$$
H=\left(\pi D_{2} n g^{0,5} \sin \gamma_{2}\right)^{2}-\left(\pi D_{1} n g^{0,5} \sin \gamma_{1}\right)^{2}+Q n g b_{2}^{-1} \sin ^{2} \gamma_{2} \cdot \operatorname{ctg} \beta_{2}-Q n g b_{1}^{-1} \sin ^{2} \gamma_{1} \cdot \operatorname{ctg} \beta_{1} .
$$

Следует учесть, что реальное количество напорных лопастей исполнительного элемента ограничено и находится в диапазоне от 3 до 48 (в зависимости от назначения ГЭК), что приводит к снижению давления по сравнению с идеализированной моделью. Приближенный учет влияния конечного числа лопастей $z$ может быть сделан с помощью коэффициента $k_{H}[5]$ :

$$
k_{H}=1-\frac{\Delta H}{H},
$$

где

$$
\Delta H=u_{2}^{2} \frac{\pi}{z}\left(1-\frac{D_{1}^{2}}{D_{2}^{2}}\right) \sin \beta_{2} .
$$

Ориентировочно значение $k_{H}$ находится в диапазоне от 0,7 до 0,9.

Задача нахождения гидравлических, объемных и механических потерь при движении охлаждающей жидкости чрезвычайно сложна и требует специального изучения закономерностей течения жидкости. Теоретически поток жидкости можно характеризовать объемным расходом и средней скоростью по сечению трубы. При движении реальных жидкостей часть 


\section{Учёные записки \\ Комсомольского-на-Амуре государственного технического университета}

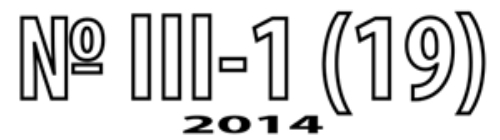

механической энергии движения переходит в тепловую и представляет потери энергии $\Delta E$, обусловленные молекулярной и турбулентной вязкостью жидкости. С потерями энергии также связаны потери давления $\Delta P$ :

$$
\Delta P=\rho \cdot \Delta E
$$

и потери напора $\Delta h$ :

$$
\Delta h=\frac{\Delta P}{\rho g}
$$

где $\rho$ - плотность жидкости.

Приближенный учет механических потерь может быть сделан по коэффициенту быстроходности $n_{s}$ :

$$
n_{S}=3,65 n \sqrt[4]{\frac{Q^{2} N^{3}}{H^{3}}},
$$

где $N$ - количество ступеней, составляющих исполнительный элемент.

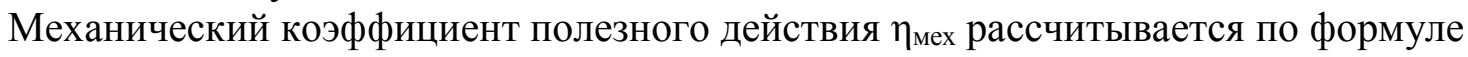

$$
\eta_{\text {мех }}=\frac{1}{1+820 n_{s}^{-2}}
$$

и находится в диапазоне от 0,92 до 0,96.

Гидравлические потери давления обычно определяют отдельно для относительно длинных участков $\Delta P_{\text {TP }}$ на которых движение жидкости достаточно равномерно, и для коротких участков $\Delta P_{\mathrm{M}}$, характеризующихся местными изменениями формы канала с возможностью образования вихрей в потоке.

Гидравлические потери давления и напора на прямых участках $\Delta P_{\mathrm{TP}}$ и $\Delta h_{\mathrm{TP}}$ могут быть рассчитаны по формулам

$$
\Delta P_{\mathrm{TP}}=\lambda \cdot \frac{\rho l}{2 d} \cdot v^{2}, \quad \Delta h_{\mathrm{TP}}=\lambda \cdot \frac{l}{2 d g} \cdot v^{2},
$$

где $\lambda$ - коэффициент гидравлического трения, зависящий от режима течения жидкости и шероховатости поверхности; $l$ - длина прямого участка канала; $d$ - диаметр прямого участка канала.

Потери давления $\Delta P_{\mathrm{M}}$ и потери напора $\Delta h_{\mathrm{M}}$ на коротких участках со сложной геометрией достаточно точно рассчитываются по формуле Вейсбаха:

$$
\Delta P_{\mathrm{M}}=\xi \cdot \frac{\eta}{v} \cdot \frac{v^{2}}{2}, \quad \Delta h_{\mathrm{M}}=\xi \cdot \frac{v^{2}}{2 g},
$$

где $\xi$ - коэффициент местного гидравлического сопротивления, зависящий от режима течения, вида и конструктивного исполнения канала; $\eta$ - динамическая вязкость; $v$ - кинематическая вязкость.

Значения коэффициентов сопротивления определяются опытным путем и в обобщенном виде приводятся в виде эмпирических формул или графиков.

Полные гидравлические потери давления и напора ( $\Delta P$ и $\Delta h$ соответственно) определяются выражениями

$$
\begin{gathered}
\Delta P=\Delta P_{\mathrm{TP}}+\Delta P_{\mathrm{M}}, \\
\Delta h=\Delta h_{\mathrm{TP}}+\Delta h_{\mathrm{M}}
\end{gathered}
$$

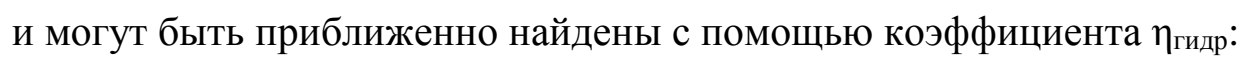




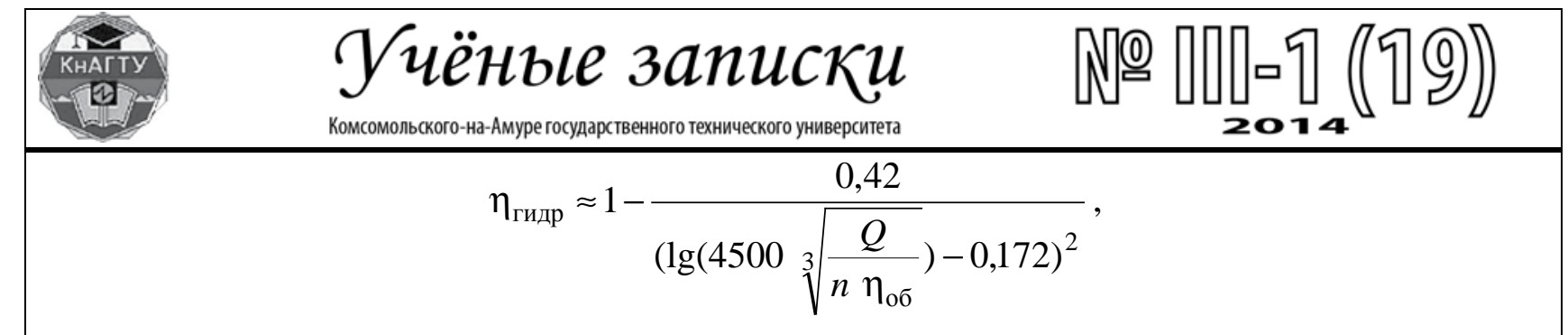

где $\eta_{\text {об }}$ объемный коэффициент полезного действия.

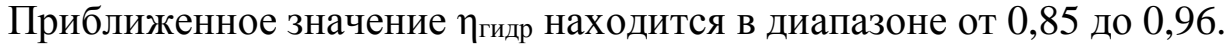

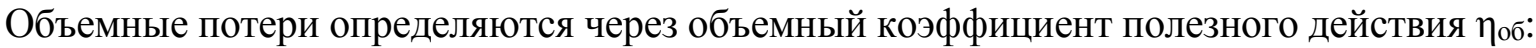

$$
\eta_{\text {об }}=\frac{1}{1+0,68 n_{S}^{-0,66}} \text {. }
$$

Коэффициент объемного сжатия находится в диапазоне от 0,85 до 0,95.

Соответственно, механическая составляющая мощности, расходуемая на транспортирование охлаждающей жидкости, может быть записана в следующем виде:

$$
P_{\text {мех }}=\frac{\rho g H_{\text {д }} Q_{\text {д }}}{k_{H} \eta_{\text {об }} \eta_{\text {гидр }} \eta_{\text {мех }}},
$$

где $H_{д}$ - действительное значение давления; $Q_{д}$ - действительное значение производительности. Указанные параметры даны с учетом конечного числа напорных лопастей и объемного сжатия теплоносителя.

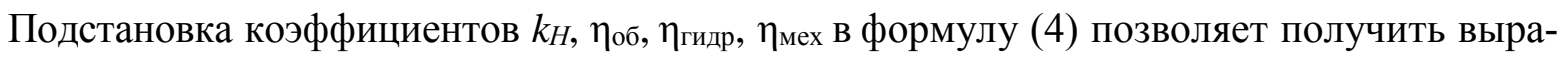
жение для первой энергетической составляющей $P_{2 \text { мех }}$ (механической) с учетом проектных и эксплуатационных параметров ГЭК:

$$
P_{2 \text { мех }}=\frac{\rho g\left(\lambda_{1}+\lambda_{2} \cdot Q\right)^{2} Q \cdot\left(1+0,68 \lambda_{3}^{-0,66} \cdot \frac{Q^{-0,33}}{\left(\lambda_{1}+\lambda_{2} \cdot Q\right)^{-0,495}}\right) \cdot\left(1+820 \cdot \lambda_{3}^{-2} \cdot\left(\lambda_{1} \cdot Q^{-\frac{2}{3}}+\lambda_{2} \cdot Q^{\frac{1}{3}}\right)^{1,5}\right)}{\left(\left(\lambda_{1}-\Delta H\right)+\lambda_{2} \cdot Q\right) \cdot k_{Q} \cdot\left(1-\frac{3,78}{\lg ^{2}\left(\lambda_{4} \cdot\left(Q+\frac{0,68 \lambda_{3}^{-0,66}}{\left(\lambda_{1}+\lambda_{2} \cdot Q\right)^{-0,495}}\right)\right)}\right)},
$$

где

$$
\begin{gathered}
\lambda_{1}=\left(\pi D_{2} n g g^{0,5} \sin \gamma_{2}\right)^{2}-\left(\pi D_{1} n g g^{0,5} \sin \gamma_{1}\right)^{2}=\pi^{2} n g\left(D_{2}^{2} \sin ^{2} \gamma_{2}-D_{1}^{2} \sin ^{2} \gamma_{1}\right), \\
\lambda_{2}=n^{2} g\left(b_{2}^{-1} \sin ^{2} \gamma_{2} \cdot \operatorname{ctg} \beta_{2}-b_{1}^{-1} \sin ^{2} \gamma_{1} \cdot \operatorname{ctg} \beta_{1}\right), \\
\lambda_{3}=3,65 n N^{0,75}, \\
\lambda_{4}=\frac{4500}{10^{0,172} n^{\frac{1}{3}}} .
\end{gathered}
$$

Вторая составляющая (тепловая) характеризует ГЭК как устройство генерации тепловой энергии и определяется результатом взаимодействия тепло- и массообменных процессов. В работе [5] приведено выражение, из которого может быть получена формула для прибли-

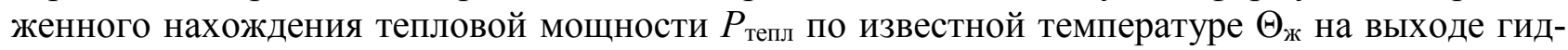
равлической цепи ГЭК: 


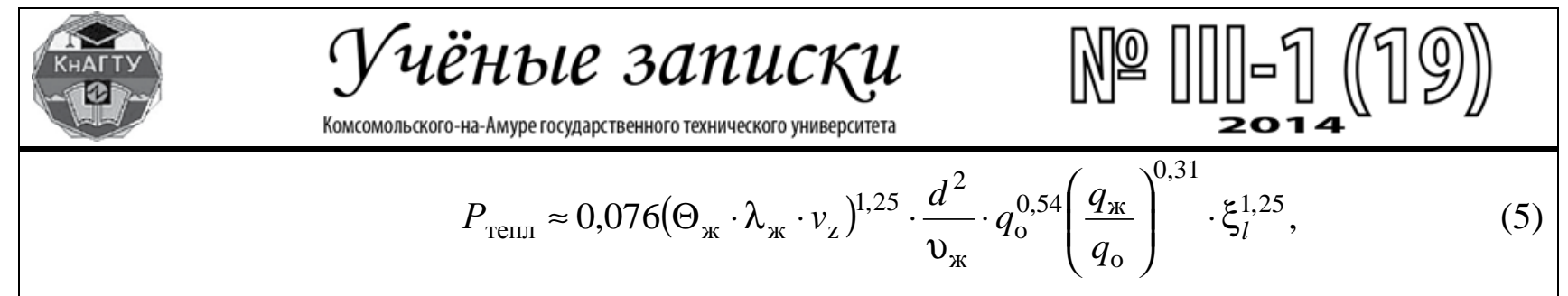

где $v, \rho, c_{p}, \lambda$ - соответственно кинематическая вязкость, плотность, удельная теплоемкость и теплопроводность охлаждающей среды, приведенные к расчетным температурам жидкости (нижний индекс «ж») или герметизирующей оболочки (нижний индекс «о»); $\xi_{l}-$ поправочный коэффициент, учитывающий отношение длины $l$ и диаметра $d$ канала.

Выражение (5) позволяет решить не только прямую задачу определения тепловой составляющей в энергетическом балансе ГЭК, но и обратную задачу - обеспечить требуемые энергетические соотношения, например за счет вариации размерных соотношений исполнительного элемента с помощью аппроксимирующих моделей осевой составляющей абсолютной скорости в канале [4].

Совместное использование выражений (4) и (5) определяет долевое соотношение между тепловой и механической мощностью и условный коэффициент тепломеханического использования, характеризующие эксплуатационный режим работы герметичного электротехнического комплекса.

\section{ЛИТЕРАТУРА}

1. Голоколос, Д. А. Оценка влияния экранирующих элементов на характеристики герметичных электромеханических преобразователей / Д. А. Голоколос, Г. М. Гринфельд // Ученые записки Комсомольского-на-Амуре государственного технического университета. Науки о природе и технике. 2013. - № I-1(13). - С. 18-25.

2. Ким, К. К. Электромеханические генераторы тепловой энергии: генерация и передача тепловой энергии : моногр. / К. К. Ким, С. Н. Иванов. - Saarbrücken, Deutschland : Издательство LAP (LAMBERT Academic Publishing). - 2011. - 352 c.

3. Иванов, С. Н. Использование электромеханических преобразователей в качестве устройств электронагрева / С. Н. Иванов // Научно-технические ведомости СПбГТУ. Основной выпуск. - 2008. № 3. - С. 246-252.

4. Исследование процессов массо- и теплообмена в совмещенных энергетических устройствах методами визуального анализа / К. К. Ким, О. В. Приходченко, А. А. Просолович // Ученые записки Комсомольского-на-Амуре государственного технического университета. Науки о природе и технике. 2014. - № I-1(16). - С. 22-29.

5. Приходченко, О. В. Математическое моделирование и анализ электромагнитных и тепловых процессов в электромеханических теплогенерирующих преобразователях / О. В. Приходченко // Ученые записки Комсомольского-на-Амуре государственного технического университета. Науки о природе и технике. - 2011. - № III-1(7). - С. 27-37. 